\title{
THE ZEROS OF THE SECOND DERIVATIVE OF THE RECIPROCAL OF AN ENTIRE FUNCTION
}

\author{
BY \\ SIMON HELLERSTEIN ${ }^{1}$ AND JACK WILLIAMSON
}

\begin{abstract}
Let $f$ be a real entire function of finite order with only real zeros. Assuming that $f^{\prime}$ has only real zeros, we show that the number of nonreal zeros of $f^{\prime \prime}$ equals the number of real zeros of $F^{\prime \prime}$, where $F=1 / f$. From this, we show that $F^{\prime \prime}$ has only real zeros if and only if $f(z)=\exp \left(a z^{2}+b z+c\right), a>0$, or $f(z)=$ $(A z+B)^{n}, A \neq 0, n$ a positive integer.
\end{abstract}

1. Introduction. The authors recently proved in [1], [2]

TheOReM A. Let $f(z)$ be a (constant multiple of a) real entire function (i.e., z real implies $f(z)$ real) with only real zeros. Assume that $f^{\prime}(z)$ has only real zeros. Then $f^{\prime \prime}(z)$ has only real zeros if and only if $f(z)$ is of the form

$$
f(z)=z^{m} e^{-a z^{2}+b z+c} \prod_{n}\left(1-z / z_{n}\right) e^{z / z_{n}}
$$

where $m$ is a nonnegative integer, $a \geqslant 0, b$ and the $z_{n}$ are real, and $\Sigma_{n} z_{n}^{-2}<\infty$.

The class of functions of this form is called the Laguerre-Pólya class and will be denoted by $U_{0}$. The "if" half of Theorem $\mathrm{A}$ was well known and is an easy consequence of the classical result of Laguerre [3] and Pólya [6] that $f \in U_{0}$ if and only if it can be uniformly approximated on compact sets in the plane by a sequence of polynomials with only real zeros.

In this note we shall prove the following, rather striking, symmetric analog of Theorem A.

THEOREM 1. Let $F(z)=1 / f(z)$ where $f(z)$ is a (constant multiple of a) real entire function of finite order with only real zeros. Assume that $F^{\prime}(z)$ (equivalently $\left.f^{\prime}(z)\right)$ has only real zeros. Then $F^{\prime \prime}(z)$ has only nonreal zeros if and only if $f \in U_{0}$.

In [1] we obtained the exact number of nonreal zeros of $f^{\prime \prime}$ for a real entire function $f$ of finite order for which $f$ and $f^{\prime}$ have only real zeros. For such $f$, we shall show here that if $F=1 / f$, the number of real zeros of $F^{\prime \prime}$ is the same as the number of nonreal zeros of $f^{\prime \prime}$-a surprising duality.

In order to state this result precisely, we recall the following notation used in [1]. For each integer $p \geqslant 0$, denote by $V_{2 p}$ the class of entire functions of the form

$$
f(z)=\exp \left(-a z^{2 p+2}\right) g(z)
$$

Received by the editors March 13, 1979 and, in revised form, February 15, 1980.

1980 Mathematics Subject Classification. Primary 30D30.

${ }^{1}$ Supported in part by NSF Grant MCS 7903017. 
where $a \geqslant 0$ and $g(z)$ is a constant multiple of a real entire function of genus $\leqslant 2 p+1$ with only real zeros. That is, $g$ is of the form

$$
g(z)=c z^{m} e^{Q(z)} \prod_{n}\left(1-z / z_{n}\right) \exp \left[z / z_{n}+\frac{1}{2}\left(z / z_{n}\right)^{2}+\cdots+\frac{1}{q}\left(z / z_{n}\right)^{q}\right]
$$

where $c$ is a constant, $Q$ a real polynomial of degree $\leqslant 2 p+1$, the $z_{n}$ are real, $\Sigma_{n}\left|z_{n}\right|^{-q-1}<\infty$, and $q \leqslant 2 p+1$. Now set $U_{0}=V_{0}$ and for $p \geqslant 1, U_{2 p}=V_{2 p}-$ $V_{2 p-2}$. We note that every real entire function of finite order with only real zeros belongs to some $U_{2 p}$ and that $U_{0}$ is the aforementioned Laguerre-Pólya class.

In [1] we showed

THEOREM B. If $f \in U_{2 p}$ and $f^{\prime}$ has only real zeros, then $f^{\prime \prime}$ has exactly $2 p$ nonreal zeros.

Here, our main result is given by

THEOREM 2. Let $f \in U_{2 p}$. If $F=1 / f$ and $F^{\prime}$ (equivalently $f^{\prime}$ ) has only real zeros, then $F^{\prime \prime}$ has exactly $2 p$ real zeros.

Clearly, Theorem 1 is a consequence of Theorem 2.

For $p=0$, Theorem 2 is elementary and is contained in the following

Proposition. Suppose $f \in U_{0}$ and $F=1 / f$. Then $f^{\prime}$ has only real zeros and, unless $f(z)=c e^{b z}, \log |F|$ (and hence $\left.|F|\right)$ is strictly convex on any interval containing no zero of $f$.

Proof. Let $f \in U_{0}$. Then an immediate consequence of the characterization of $U_{0}$ given by Laguerre and Pólya (mentioned previously) is that $f^{\prime} \in U_{0}$ and therefore has only real zeros. Now let $I$ be a real interval containing no zero of $f$. We assume $f>0$ on $I$ (otherwise consider $-f$ ). Then $(\log F)^{\prime \prime}=(-\log f)^{\prime \prime}=$ $-\left(f^{\prime} / f\right)^{\prime}$. Since, as is well known, $\left(f^{\prime} / f\right)^{\prime}(x) \leqslant 0$ for all $x$ in $I$ (with equality holding if and only if $f(z)=c e^{b z}, b$ a real constant), the proof is complete.

We remark that when $F$ is the gamma function $\Gamma$, this is the usual proof of the log-convexity of $|\Gamma|$ on real intervals of continuity of $\Gamma$.

We shall also prove

THEOREM 3. Let $F=1 / f$, where $f$ is a real entire function of finite order with only real zeros. Suppose $F^{\prime}$ (equivalently $f^{\prime}$ ) has only real zeros. Then

(a) $F^{\prime \prime}$ has only real zeros if and only if $f(z)=e^{a z^{2}+b z+c}, a>0$, or $f(z)=$ $(A z+B)^{n}, A \neq 0$, for some positive integer $n$.

(b) If $f$ has infinitely many zeros, then $F^{\prime \prime}$ has infinitely many nonreal zeros.

The "if" part of (a) is clear. For the converse, assume that $f$ has finitely many zeros. Then since $f \in U_{2 p}$ for some $p$, we have $f(z)=P(z) e^{Q(z)}$ where $P$ and $Q$ are polynomials, and the degree of $Q$ is $2 p, 2 p+1$, or $2 p+2$. Then it is easily verified that $F^{\prime \prime}$ has more than $2 p$ zeros unless $p \leqslant 1$ and $f(z)=e^{a z^{2}+b z+c}$ or $f(z)=$ $(A z+B)^{n}, A \neq 0, n$ a positive integer. In the former case $F^{\prime \prime}$ has only real zeros only if $a \geqslant 0$. Thus in view of Theorem 2, in order to prove Theorem 3 it will be sufficient to prove part (b) of the theorem. Since Theorem 1 also follows from 
Theorem 2, the remainder of this paper is devoted to the proofs of Theorem 2 and Theorem 3(b).

2. Preliminaries. Let $f$ be a (constant multiple of a) real entire function with only real zeros. Assume that $f^{\prime}$ has only real zeros. Many of the ideas used in the proofs of Theorems 2 and 3 are found in [1] and [2]. We summarize these and set the stage for the proofs to appear in $\S \S 3$ and 4.

Without loss of generality we assume throughout that $f(0) \neq 0$ and $f^{\prime}(0) \neq 0$. Denote by $\left\{a_{n}\right\}$ the distinct zeros of $f$ and enumerate them as follows:

$$
\cdots<a_{k-1}<a_{k}<a_{k-1}<\cdots(-\infty \leqslant \alpha \leqslant k \leqslant \omega \leqslant+\infty, k \text { finite }) .
$$

According to Rolle's Theorem, $f^{\prime}$ has at least one zero in each interval $\left(a_{k}, a_{k+1}\right)$; choose exactly one and denote it by $b_{k}$ so that we have (reindexing if necessary)

$$
a_{k}<b_{k}<a_{k+1} \text { for all } k \text { with } b_{-1}<0<a_{1} \text {. }
$$

Now set

$$
\psi(z)= \begin{cases}\frac{z-b_{0}}{z-a_{0}} \prod_{k \neq 0} \frac{1-z / b_{k}}{1-z / a_{k}} & \text { if } \omega=+\infty, \\ \frac{z-b_{0}}{\left(z-a_{0}\right)\left(a_{\omega}-z\right)} \prod_{k \neq 0, \omega} \frac{1-z / b_{k}}{1-z / a_{k}} & \text { if } \omega<+\infty^{2}\end{cases}
$$

and note, as in [1], that $\psi$ is meromorphic and maps the upper half-plane into the upper half-plane. This follows from the interlacing property (2.2) (cf. [4, pp. 308-309]).

We have, therefore

$$
\frac{f^{\prime}}{f}(z)=\phi(z) \psi(z)
$$

where $\phi$ is real entire and has only real zeros. Using the growth estimates of Carathéodory $[4$, p. 18] implied by the mapping property of $\psi$ together with some estimates from Nevanlinna's theory of meromorphic functions, in particular Nevanlinna's lemma on the logarithmic derivative, we find that if $f$ is of finite order (the case considered here), $\phi$ is a polynomial (cf. [1 Lemma 2], [5, pp. 335-336]). Thus, for $f \in U_{2 p}$, we can write

$$
\frac{f^{\prime}}{f}(z)=P(z) \psi(z)
$$

where $\psi$ is given by (2.3) and $P(z)$ is a polynomial. We shall call the zeros of $P(z)$ extraordinary zeros of $f^{\prime}$ and shall call the other zeros of $f^{\prime}$-those $b_{k}$ whose multiplicity equals 1 and those $a_{k}$ whose multiplicity exceeds 1 -ordinary zeros of $f^{\prime}$.

If we denote by $\left\{\gamma_{n}\right\}_{n=\alpha^{\prime}}^{\omega^{\prime}}$ the sequence of distinct zeros of $f^{\prime} / f$, then by (2.3) and (2.5) we see that this sequence is made up of the $b_{k}$ 's and the distinct zeros of $P(z)$ (which are real in view of our assumption that $f^{\prime}$ has only real zeros) not contained

\footnotetext{
${ }^{2}$ If $f$ has only one zero, set $\psi(z)=\left(a_{0}-z\right)^{-1}$; if $f$ has no zeros, set $\psi(z) \equiv 1$.
} 
in $\left\{b_{k}\right\}$. For convenience we set

$$
\gamma_{\alpha^{\prime}-1}=-\infty \text { if }-\infty<\alpha^{\prime} \text { and } \gamma_{\omega^{\prime}+1}=+\infty \text { if } \omega^{\prime}<+\infty
$$

Now observe that for any fixed $n$ either

(i) $\left(\gamma_{n}, \gamma_{n+1}\right)$ contains exactly one zero $a_{k}$ of $f$ with multiplicity $m_{k}$ or

(ii) $\left(\gamma_{n}, \gamma_{n+1}\right)$ contains no zero of $f$.

We shall call the interval $\left(\gamma_{n}, \gamma_{n+1}\right)$ typical in case (i) and shall call it atypical in case (ii). If $f$ has no zeros, then each distinct zero of $P(z)$ determines an atypical interval; if $f$ has zeros, each distinct zero of $P(z)$ which is different from a $b_{k}$ determines an atypical interval. In this latter case, it follows that the number of atypical intervals of zeros of $f^{\prime} / f$ does not exceed the degree of $P(z)$. (Note that if $-\infty<\alpha^{\prime}$ or if $\omega^{\prime}<+\infty$, it is possible that $f^{\prime} / f$ has semi-infinite atypical intervals.) In an earlier work [1, Lemma 8] we were able to determine the degree of $P(z)$; we restate that result now as

LeMMA 1. Let $f \in U_{2 p}$. If $f$ has an infinite number of zeros, then $P(z)$ is of degree $2 p$ or $2 p+1$. If $f$ has a finite number of zeros and at least one, then $P(z)$ is of degree equal to the genus of $f$ (i.e., is of degree $2 p, 2 p+1$, or $2 p+2$ ).

Before turning to the proofs of Theorems 2 and 3 we need some technical lemmas on the growth properties of entire functions and of the function $\psi(z)$.

LemMA 2. Let $\Pi(z)$ be a canonical product of genus $p$ with only real zeros, let $\varepsilon>0$, and let

$$
\mathscr{D}=\left\{z=r e^{i \theta}: r>0 \text { and } \varepsilon \leqslant|\theta| \leqslant \pi-\varepsilon\right\} \text {. }
$$

Then

$$
\left|\frac{\Pi^{\prime}}{\Pi}(z)\right|=o\left(r^{p}\right) \quad(|z|=r \rightarrow+\infty, z \in \mathscr{D}) .
$$

LEMMA 3. Let $g(z)$ be an entire function of genus $\sigma \geqslant 2$ with only real zeros. Let $M=[\sigma / 2], \varepsilon=\pi / 2(\sigma+1), N$ be any positive integer, and let $\mathscr{D}$ be as in (2.7). Then there are at least $M$ rays $r e^{i \psi_{1}}, \ldots, r e^{i \psi_{M}}(0<r<\infty)$ in $\mathscr{D}$ such that for $k=1, \ldots, M$

$$
\left|g\left(r e^{i \psi_{k}}\right)\right|=o\left(r^{-N}\right) \quad(r \rightarrow \infty)
$$

LEMMA 4. Let $g(z)$ be a real entire function of genus $\sigma=0$ or 1 with only real zeros. If $g$ has an infinite number of zeros, then

$$
\liminf _{y \rightarrow \infty}|g( \pm i y)||y|^{-N}=\infty
$$

for any positive integer $N$.

LEMMA 5. Let $\psi(z)$ be the meromorphic function given by (2.3). Then, for $\operatorname{Im} z=y$ $\neq 0$,

$$
\left|\psi^{\prime}(z) / \operatorname{Im} \psi(z)\right| \leqslant 1 /|y|
$$


The proof of Lemma 2 is by an easy growth argument, while the proofs of Lemmas 3 and 4 follow by using the Hadamard representation to write $g=e^{Q} \Pi$ $=e^{Q} \Pi_{1} \Pi_{2}$, where $Q$ is a polynomial and $\Pi_{1}$ and $\Pi_{2}$ are the products of the positive and negative zeros of $f$ respectively. Then, in the proof of Lemma 3, we use the Valiron representation [7, p. 237] for $\log \left|\Pi_{1}\right|$ and $\log \left|\Pi_{2}\right|$ to find rays along which $\log |g|$ (and hence $|g|$ ) has the appropriate asymptotic behavior. In the proof of Lemma 4, an examination of $\log \left|\Pi_{k}( \pm i y)\right|, k=1,2$, yields the appropriate growth for $\log |g( \pm i y)|$ (and hence $|g( \pm i y)|)$.

The proof of Lemma 5, for $y>0$, follows by applying Schwarz's lemma to the function $T_{1} \psi T_{2}^{-1}$ where $T_{1}$ and $T_{2}$ are appropriate linear fractional transformations from the upper half plane onto the unit disk. A similar proof gives the result for $y<0$.

3. The proof of Theorem 2. We assume that

$$
F(z)=1 / f(z)
$$

where $f \in U_{2 p}$. We also will assume that $p \geqslant 1$, since the case $p=0$ was treated in the introduction. If $f$ has no zeros; i.e., if $f=e^{Q}$ for some polynomial $Q$, then $F$ is entire. If, in addition, $f^{\prime}$ has only real zeros, an application of Theorem B to $F$ and a simple counting argument show that $F^{\prime \prime}$ has exactly $2 p$ real zeros. Hence, in the remainder of this section we will assume that $f$ has at least one zero.

Using the notation and terminology introduced in $\S 2$, we have, by (3.1) and (2.5), that

$$
F^{\prime}(z)=-\frac{1}{f(z)} \cdot \frac{f^{\prime}}{f}(z)=-\frac{1}{f(z)} \cdot P(z) \psi(z)
$$

where, by assumption, $F^{\prime}\left(f^{\prime}\right)$ has only real zeros. Since (3.2) shows that the zeros of $F^{\prime}$ correspond to the zeros of $f^{\prime} / f$-the $\gamma_{n}$ 's-we will prove Theorem 2 by first showing that in a typical interval of zeros of $f^{\prime} / f, F^{\prime \prime}$ has no zeros and in a finite (an infinite) atypical interval of zeros of $f^{\prime} / f, F^{\prime \prime}$ has a (at most a) single zero of multiplicity one. From this it will follow that the number of real zeros of $F^{\prime \prime}$ is equal to the degree of $P(z)$ minus the number of semi-infinite atypical intervals of $f^{\prime} / f$ in which $F^{\prime \prime}$ has no zeros. We will then determine the precise number of zeros of $F^{\prime \prime}$ in any semi-infinite atypical interval of $f^{\prime} / f$ and thereby conclude that $F^{\prime \prime}$ has exactly $2 p$ real zeros.

Suppose then that $\left(\gamma_{n}, \gamma_{n+1}\right)$ is a typical interval of zeros of $f^{\prime} / f$ so that $\left(\gamma_{n}, \gamma_{n+1}\right)$ contains exactly one zero $a_{k}$ of $f$ of multiplicity $m_{k}$. Assume in what follows that $\left(\gamma_{n}, \gamma_{n+1}\right)$ is a finite interval (the analysis is similar to that which follows if $\left(\gamma_{n}\right.$, $\left.\gamma_{n+1}\right)$ is a semi-infinite interval). Then each of the intervals $\left(\gamma_{n}, a_{k}\right),\left(a_{k}, \gamma_{n+1}\right)$ contains an even number of zeros of $F^{\prime \prime}$. To see this observe that since $\gamma_{n}$ is a zero of $F^{\prime}$, it follows that in some neighborhood $\eta$ of $\gamma_{n}$,

$$
F^{\prime}(z)=\left(z-\gamma_{n}\right)^{l} h(z)
$$

where $l \geqslant 1, h(z)$ is holomorphic in $\eta$, and $h\left(\gamma_{n}\right) \neq 0$. From this it follows that

$$
\frac{F^{\prime \prime}}{F^{\prime}}(z)=\frac{l}{z-\gamma_{n}}+\frac{h^{\prime}}{h}(z) \quad\left(z \in \eta, z \neq \gamma_{n}\right)
$$


and hence that

$$
\lim _{x \rightarrow \gamma_{n}^{+}} \frac{F^{\prime \prime}}{F^{\prime}}(x)=+\infty \quad \text { for } \operatorname{Re} z=x \in \eta .
$$

On the other hand, since $a_{k}$ is a zero of $f$ and therefore by (3.2) a pole of $F^{\prime}$, a similar analysis shows that

$$
\lim _{x \rightarrow a_{\bar{k}}^{-}} \frac{F^{\prime \prime}}{F^{\prime}}(x)=+\infty \text {. }
$$

Thus, since $F^{\prime \prime}$ is analytic on $\left(\gamma_{k}, a_{k}\right),(3.5)$ and (3.6) imply that $F^{\prime \prime}$ has an even number of zeros in $\left(\gamma_{n}, a_{k}\right)$. The same analysis yields the same conclusion for the interval $\left(a_{k}, \gamma_{n+1}\right)$.

We will now show that $F^{\prime \prime}$ can have at most one simple zero in each of the intervals $\left(\gamma_{n}, a_{k}\right),\left(a_{k}, \gamma_{n+1}\right)$. In view of the above, it will then follow that $F^{\prime \prime}$ has no zeros in the typical interval $\left(\gamma_{n}, \gamma_{n+1}\right)$. To do this we use the representation (3.2) and set

$$
H_{k}(z)= \begin{cases}\left(z-a_{k}\right) P(z) \psi(z)=-\left(z-a_{k}\right) \frac{F^{\prime}}{F}(z), & z \neq a_{k}, \\ m_{k}, & z=a_{k} .\end{cases}
$$

Then, in view of (3.7), we can write

$$
\frac{F^{\prime \prime}}{F^{\prime}}(z)=\frac{H_{k}^{\prime}}{H_{k}}(z)-\frac{1}{z-a_{k}}-\frac{H_{k}(z)}{z-a_{k}} .
$$

Now we will analyze the function $H_{k}(x)$ for $\gamma_{n}<x=\operatorname{Re} z<\gamma_{n+1}$. Since we are assuming $\left(\gamma_{n}, \gamma_{n+1}\right)$ is a finite interval, we observe that

$$
\begin{gathered}
H_{k}(x) \text { is analytic on }\left[\gamma_{n}, \gamma_{n+1}\right], \\
H_{k}\left(\gamma_{n}\right)=H_{k}\left(\gamma_{n+1}\right)=0,
\end{gathered}
$$

and

$$
H_{k}(x) \neq 0 \text { on }\left(\gamma_{n}, \gamma_{n+1}\right)
$$

Consequently, since

$$
H_{k}\left(a_{k}\right)=m_{k}>1
$$

(3.9), (3.11), and (3.12) imply that

$$
H_{k}(x)>0 \text { for } \gamma_{n}<x<\gamma_{n+1} \text {. }
$$

Repeating the arguments used in the proof of Lemma 7 of [1], we find that

$$
\left[\frac{H_{k}^{\prime}}{H_{k}}(x)\right]^{\prime}<0 \text { for } \gamma_{n}<x<\gamma_{n+1}
$$

and that there exists $x_{0} \in\left(\gamma_{n}, \gamma_{n+1}\right)$ such that

$$
H_{k}^{\prime}(x) \begin{cases}>0 & \text { for } \gamma_{n}<x<x_{0} \\ =0 & \text { for } x=x_{0} \\ <0 & \text { for } x_{0}<x<\gamma_{n+1}\end{cases}
$$


Thus (3.13) and (3.15) imply that

$$
\frac{H_{k}^{\prime}}{H_{k}}(x) \begin{cases}>0 & \text { for } \gamma_{n}<x<x_{0} \\ =0 & \text { for } x=x_{0} \\ <0 & \text { for } x_{0}<x<\gamma_{n+1}\end{cases}
$$

Now suppose that

$$
\gamma_{n}<x_{0}<a_{k} .
$$

(In case $a_{k}<x_{0}<\gamma_{n+1}$ or $x_{0}=a_{k}$, the analysis is similar to that which follows.) Then (3.8), (3.13), (3.16), and (3.17) imply that

$$
\begin{aligned}
\left(x-a_{k}\right) \frac{F^{\prime \prime}}{F^{\prime}}(x) & =\left(x-a_{k}\right) \frac{H_{k}^{\prime}}{H_{k}}(x)-1-H_{k}(x) \\
& <0 \text { for } a_{k}<x<\gamma_{n+1} .
\end{aligned}
$$

Thus, (3.18) implies that $F^{\prime \prime}$ has no zero on $\left(a_{k}, \gamma_{n+1}\right)$.

An analysis similar to that which led to (3.18) shows that

$$
\left(x-a_{k}\right) \frac{F^{\prime \prime}}{F^{\prime}}(x)<0 \text { for } \gamma_{n}<x \leqslant x_{0} .
$$

Next, differentiating (3.18) we obtain

$$
\begin{aligned}
{\left[\left(x-a_{k}\right) \frac{F^{\prime \prime}}{F^{\prime}}(x)\right]^{\prime} } & =\left(x-a_{k}\right)\left[\frac{H_{k}^{\prime}}{H_{k}}(x)\right]^{\prime}+\frac{H_{k}^{\prime}}{H_{k}}(x)-H_{k}^{\prime}(x) \\
& =\left(x-a_{k}\right)\left[\frac{H_{k}^{\prime}}{H_{k}}(x)\right]^{\prime}+\left[1-H_{k}(x)\right] \cdot \frac{H_{k}^{\prime}}{H_{k}}(x) .
\end{aligned}
$$

Since (3.15) implies that

$$
H_{k}(x)>H_{k}\left(a_{k}\right)=m_{k} \geqslant 1 \text { for } x_{0}<x<a_{k},
$$

it follows from (3.14), (3.16), (3.17), (3.20), and (3.21) that

$$
\left[\left(x-a_{k}\right) \frac{F^{\prime \prime}}{F^{\prime}}(x)\right]^{\prime}>0 \text { for } x_{0}<x<a_{k} .
$$

Thus, (3.19) and (3.22) imply that $F^{\prime \prime}$ has at most one simple zero on $\left(\gamma_{n}, a_{k}\right)$.

This completes the proof that $F^{\prime \prime}$ has no zeros in a typical interval.

Suppose now that the interval $\left(\gamma_{n}, \gamma_{n+1}\right)$ is an atypical interval of zeros of $f^{\prime}$; that is, suppose that it contains no zero of $f$. We will show that if it is a finite interval, it contains exactly one simple zero of $F^{\prime \prime}$ and that if it is a semi-infinite interval it contains at most one simple zero of $F^{\prime \prime}$. Observe that in the former case $\gamma_{n}$ and $\gamma_{n+1}$ are zeros of $F^{\prime}$; hence, the analysis in (3.3) - (3.5) shows that

$$
\lim _{x \rightarrow \gamma_{n}^{+}} \frac{F^{\prime \prime}}{F^{\prime}}(x)=+\infty
$$

while

$$
\lim _{x \rightarrow \gamma_{n+1}^{-}} \frac{F^{\prime \prime}}{F^{\prime}}(x)=-\infty
$$

Thus, in case $\left(\gamma_{n}, \gamma_{n+1}\right)$ is a finite interval, $F^{\prime \prime}$ has at least one zero in $\left(\gamma_{n}, \gamma_{n+1}\right)$. Clearly then, we will be done if we can show that $F^{\prime \prime}$ has at most one simple zero in 
the finite or infinite interval $\left(\gamma_{n}, \gamma_{n+1}\right)$. To do this we set

$$
H(z)=P(z) \psi(z)=-\frac{F^{\prime}}{F}(z) \text {. }
$$

Thus,

$$
\frac{F^{\prime \prime}}{F^{\prime}}(z)=\frac{H^{\prime}}{H}(z)-H(z) .
$$

As before, we analyze the function $H(x)$ on the interval $\gamma_{n}<x=\operatorname{Re} z<\gamma_{n+1}$. We will assume, also as before, that $\left(\gamma_{n}, \gamma_{n+1}\right)$ is a finite interval since the analysis is similar to that which follows if $\left(\gamma_{n}, \gamma_{n+1}\right)$ is a semi-infinite interval.

Now

$$
\begin{gathered}
H(x) \text { is analytic on }\left[\gamma_{n}, \gamma_{n+1}\right] \\
H\left(\gamma_{n}\right)=H\left(\gamma_{n+1}\right)=0
\end{gathered}
$$

and

$$
H(x) \neq 0 \text { for } \gamma_{n}<x<\gamma_{n+1} \text {. }
$$

thus (3.27) and (3.29) imply that $H(x)$ is of constant sign on $\left(\gamma_{n}, \gamma_{n+1}\right)$, say

$$
H(x)>0 \text { on }\left(\gamma_{n}, \gamma_{n+1}\right) \text {. }
$$

(The analysis is similar to that which follows if $H(x)<0$ on $\left(\gamma_{n}, \gamma_{n+1}\right)$.)

Again, it follows from arguments used in the proof of Lemma 7 of [1] that

$$
\left[\frac{H^{\prime}}{H}(x)\right]^{\prime}<0 \text { for } \gamma_{n}<x<\gamma_{n+1}
$$

and that there exists $x_{0} \in\left(\gamma_{n}, \gamma_{n+1}\right)$ such that

$$
H^{\prime}(x) \begin{cases}>0 & \text { for } \gamma_{n}<x<x_{0} \\ =0 & \text { for } x=x_{0} \\ <0 & \text { for } x_{0}<x<\gamma_{n+1}\end{cases}
$$

Thus (3.30) and (3.32) imply that

$$
\frac{H^{\prime}}{H}(x) \begin{cases}>0 & \text { for } \gamma_{n}<x<x_{0} \\ =0 & \text { for } x=x_{0} \\ <0 & \text { for } x_{0}<x<\gamma_{n+1}\end{cases}
$$

It now follows from (3.26) and (3.30)-(3.33) that

$$
\left[\frac{F^{\prime \prime}}{F^{\prime}}(x)\right]^{\prime}=\left[\frac{H^{\prime}}{H}(x)\right]^{\prime}-H^{\prime}(x)<0 \text { for } \gamma_{n}<x<x_{0}
$$

and that

$$
\frac{F^{\prime \prime}}{F^{\prime}}(x)=\frac{H^{\prime}}{H}(x)-H(x)<0 \text { for } x_{0} \leqslant x<\gamma_{n+1} \text {. }
$$

Clearly then, (3.34) and (3.35) imply that $F^{\prime \prime}$ has at most one simple zero in $\left(\gamma_{n}, \gamma_{n+1}\right)$.

In summary, the foregoing analysis together with (3.2) shows that real zeros of $F^{\prime \prime}$ can arise only from atypical intervals of zeros of $f^{\prime} / f$ or from multiple zeros of $f^{\prime} / f$. Precisely: the exact number of real zeros of $F^{\prime \prime}$ is equal to the number of 
atypical intervals of $f^{\prime} / f$ plus $\Sigma_{n}\left(l_{n}-1\right)$ minus the number of semi-infinite atypical intervals of $f^{\prime} / f$ in which $F^{\prime \prime}$ has no zeros. Here $l_{n}=$ multiplicity of $\gamma_{n}$; the sum is finite since by (2.3) and (2.5) $l_{n}=1$ for all but a finite number of $\gamma_{n}$. Since each distinct zero of $P(z)$ which is different from a $b_{k}$ determines an atypical interval and since by (2.3) and (2.5) every $b_{k}$ which is also a zero of $P(z)$ is multiple zero of $f^{\prime} / f$, it follows that the exact number of real zeros of $F^{\prime \prime}$ equals the degree of $P(z)$ minus the number of semi-infinite atypical intervals of $f^{\prime} / f$ in which $F^{\prime \prime}$ has no zeros.

To determine whether a semi-infinite atypical interval of $f^{\prime} / f$ contains a zero of $F^{\prime \prime}$, suppose that $\omega<+\infty$ and that $a_{\omega}<\gamma_{\omega^{\prime}}<+\infty$, where, recall, $\gamma_{\omega^{\prime}}$ denotes the largest zero of $f^{\prime} / f$ (i.e., in this situation, of $P$ ).

Then, $\left(\gamma_{\omega^{\prime}},+\infty\right)$ is a semi-infinite atypical interval of $f^{\prime} / f$ and by (3.23), with $\gamma_{n}$ replaced by $\gamma_{\omega^{\prime}}$,

$$
\lim _{x \rightarrow \gamma_{\omega^{+}}^{+}} \frac{F^{\prime \prime}}{F^{\prime}}(x)=+\infty .
$$

To determine whether $F^{\prime \prime}$ has one or no zero in $\left(\gamma_{\omega^{\prime}},+\infty\right)$ we will use (3.36) and the behavior of $\left(F^{\prime \prime} / F^{\prime}\right)(x)$ at $+\infty$. To obtain the latter, note that (3.2) implies

$$
\frac{F^{\prime \prime}}{F^{\prime}}=\frac{\left(f^{\prime} / f\right)^{\prime}}{f^{\prime} / f}-\frac{f^{\prime}}{f}
$$

and thus, by (2.5),

$$
\frac{F^{\prime \prime}}{F^{\prime}}=\frac{P^{\prime}}{P}+\frac{\psi^{\prime}}{\psi}-\frac{f^{\prime}}{f}
$$

Now

$$
\frac{P^{\prime}}{P}(x)=o(1)
$$

and (2.3) easily implies

$$
\frac{\psi^{\prime}}{\psi}(x)=O(1) \quad(x \rightarrow+\infty, \omega<+\infty) .
$$

Hence (3.38) and (3.39) imply that for $\omega<+\infty$ the behavior of $\left(F^{\prime \prime} / F^{\prime}\right)(x)$ at $+\infty$ is determined by the behavior of $\left(f^{\prime} / f\right)(x)$ at $+\infty$. To see what this is, first note that it is not hard to deduce from (2.3) that for $\omega<+\infty$

$$
|\psi(x)| \geqslant A / x \quad\left(x \geqslant x_{0}>\left|a_{\omega}\right|\right)
$$

where $A$ is positive constant. Further, since we are assuming $p \geqslant 1$, Lemma 1 implies

$$
|P(x)| \geqslant B x^{2} \quad\left(x \geqslant x_{1}\right)
$$

for some positive constant $B$. Thus, (2.5), (3.40), and (3.41) imply

$$
\lim _{x \rightarrow+\infty} \frac{f^{\prime}}{f}(x)=+\infty \quad(\omega<+\infty)
$$

or

$$
\lim _{x \rightarrow+\infty} \frac{f^{\prime}}{f}(x)=-\infty \quad(\omega<+\infty) .
$$


If (3.42) holds then (3.37) - (3.39) imply

$$
\lim _{x \rightarrow+\infty} \frac{F^{\prime \prime}}{F^{\prime}}(x)=-\infty \text {. }
$$

Because of (3.36), (3.44), and the behavior of $F^{\prime \prime}$ in atypical intervals, it follows that if (3.42) holds $F^{\prime \prime}$ has exactly one (simple) zero in the atypical interval $\left(\gamma_{\omega^{\prime}},+\infty\right)$. On the other hand, if (3.43) holds then (3.37)-(3.39) imply

$$
\lim _{x \rightarrow+\infty} \frac{F^{\prime \prime}}{F^{\prime}}(x)=+\infty \text {. }
$$

Hence (3.36) and (3.45) imply that if (3.43) holds $F^{\prime \prime}$ has no zeros in the atypical interval $\left(\gamma_{\omega^{\prime}},+\infty\right)$. With a change of variable from $x$ to $-x$ analogous conclusions can be drawn if $-\infty<\gamma_{\alpha^{\prime}}<a_{\alpha}$.

To complete the proof of Theorem 2 we consider separately the cases where $f$ has an infinite number of zeros and where it has a finite number of zeros. We will use the previous remarks to show that in each case $F^{\prime \prime}$ has exactly $2 p$ real zeros. If $f$ has an infinite number of zeros, then by Lemma $1 P(z)$ is of degree $2 p$ or $2 p+1$. The proof of this lemma (see [1, equation (3.57)]) shows that

$$
P\left(a_{k}\right)<0, \quad \alpha \leqslant k \leqslant \omega .
$$

Thus it is clear that if $f$ has an infinite number of positive zeros and an infinite number of negative zeros then degree $P(z)=2 p$, and therefore $F^{\prime \prime}$ has exactly $2 p$ real zeros.

Now suppose that $f$ has a finite number of zeros (or perpaps none at all) on one of the axes, say the positive axis (the analysis is similar to that which follows in the other situation). To show that $F^{\prime \prime}$ has exactly $2 p$ real zeros in this case also, we need to make several observations. First,

$$
\lim _{x \rightarrow a_{\omega}^{+}} \frac{f^{\prime}}{f}(x)=+\infty
$$

If (3.42) also holds, then (2.3), (2.5), and (3.47) imply that $P(z)$ has an even number of zeros in $\left(a_{\omega},+\infty\right)$; hence, by (3.46) $P(z)$ is of even degree; i.e., of degree $2 p$. If $P(z)$ has no zeros in $\left(a_{\omega},+\infty\right)$, then it follows that $F^{\prime \prime}$ has exactly $2 p$ real zeros. On the other hand, if $P(z)$ has some zeros in $\left(a_{\omega},+\infty\right)$, then $f^{\prime} / f$ has an infinite atypical interval in which, by the remark following (3.44), $F^{\prime \prime}$ has a simple zero. Thus $F^{\prime \prime}$ has exactly $2 p$ real zeros. On the other hand, if (3.43) holds instead of (3.42), then (2.3), (2.5), and (3.47) imply that $P(z)$ has an odd number of zeros in $\left(a_{\omega},+\infty\right)$. Hence $f^{\prime} / f$ will have an infinite atypical interval and, by (3.46), $P(z)$ is of degree $2 p+1$. But then, by the remark following (3.45), $F^{\prime \prime}$ has no zeros in this infinite atypical interval; consequently, $F^{\prime \prime}$ has exactly $2 p+1-1=2 p$ real zeros.

In the case that $f$ has a finite number of zeros,

$$
f(z)=e^{Q(z)} \pi(z)
$$

where $\pi(z)$ is a polynomial and where

$$
Q(z)=-a z^{2 p+2}+b z^{2 p+1}+c z^{2 p}+\cdots, \quad a \geqslant 0 .
$$


Since $f \in U_{2 p}$,

$$
a^{2}+b^{2}+c^{2}>0 \text { and } c>0 \text { if } a=b=0 .
$$

Since each of the three cases $a>0 ; a=0, b \neq 0 ; a=b=0, c>0$ is similarly handled, we will treat only the case $a>0$. If $a>0$, then $f$ is of genus $2 p+2$, and, by Lemma $1, P(z)$ is of degree $2 p+2$. Since (3.48) and (3.49) imply

$$
\begin{aligned}
\frac{f^{\prime}}{f}(z)= & -a(2 p+2) z^{2 p+1}+b(2 p+1) z^{2 p}+2 c p z^{2 p-1}+\cdots \\
& +\sum_{k=\alpha}^{\omega} \frac{m_{k}}{z-a_{k}} \quad(a>0)
\end{aligned}
$$

it is clear that

$$
\lim _{x \rightarrow a_{\omega}^{+}} \frac{f^{\prime}}{f}(x)=+\infty ; \quad \lim _{x \rightarrow+\infty} \frac{f^{\prime}}{f}(x)=-\infty
$$

and

$$
\lim _{x \rightarrow a_{\alpha}^{-}} \frac{f^{\prime}}{f}(x)=-\infty ; \quad \lim _{x \rightarrow-\infty} \frac{f^{\prime}}{f}(x)=+\infty ;
$$

hence, (2.5) implies that $P(z)$ has an odd number of zeros in each of the intervals $\left(-\infty, a_{\alpha}\right),\left(a_{\omega},+\infty\right)$. Thus, if $a>0, f^{\prime} / f$ has 2 semi-infinite atypical intervals. Since the last equation in (3.52) corresponds to (3.43) and the last equation in (3.53) is the analogue of (3.43) for the atypical interval $\left(-\infty, \gamma_{\alpha^{\prime}}\right)$, it follows from the remarks following (3.45) that $F^{\prime \prime}$ has no zeros in each of the 2 semi-infinite atypical intervals of $f^{\prime} / f$. Thus $F^{\prime \prime}$ has exactly $2 p+2-2=2 p$ real zeros. Since, as we remarked, the other two cases $a=0, b \neq 0 ; a=b=0, c>0$, are similarly handled, it follows that if $f$ has a finite number of zeros, $F^{\prime \prime}$ has exactly $2 p$ real zeros.

This completes the proof of Theorem 2 .

4. The proof of Theorem 3(b). As we indicated in the Introduction the idea behind the proof is the following: Since Theorem 2 tells us that $F^{\prime \prime}$ has a finite number of real zeros, it is sufficient to show that if $f$ has an infinite number of zeros then the same is true of $F^{\prime \prime}$. To do this, we observe that on the one hand by (3.37),

$$
\frac{F^{\prime \prime}}{F^{\prime}}(z)=\frac{P^{\prime}}{P}(z)+\frac{\psi^{\prime}}{\psi}(z)-\frac{f^{\prime}}{f}(z) .
$$

On the other hand, if $F^{\prime \prime}$ has only finitely many zeros, then

$$
\frac{F^{\prime \prime}}{F^{\prime}}(z)=\frac{s(z)}{g(z)}
$$

where $s(z)$ is a polynomial and $g(z)$ is a real entire function of finite order with only real zeros; indeed, $g(z)$ will have infinitely many zeros in view of the hypothesis on $f$. We will use the growth properties expressed in Lemmas 2-5 to show that (4.1) and (4.2) are incompatible.

To obtain the contradiction between (4.1) and (4.2) we assume $f \in U_{2 p}$ for some $p$ and treat separately the cases that $g(z)$ has genus $\sigma \geq 2$ and has genus $\sigma=0$ or 1 . 
We first consider the case that $\sigma \geqslant 2$. On the one hand, (4.2) and Lemma 3 imply that if $\mathscr{D}$ is as in (2.7), then there are [o/2] rays $r e^{i \psi_{j}} \in \mathscr{D}$ such that

$$
\left|\frac{F^{\prime \prime}}{F^{\prime}}\left(r e^{i \psi_{j}}\right)\right| \geqslant r^{2 p+2} \quad\left(r \geqslant r_{0}, j=1,2, \ldots,[\sigma / 2]\right) .
$$

On the other hand, turning to (4.1) we see that

$$
\left|\frac{F^{\prime \prime}}{F^{\prime}}(z)\right| \leqslant\left|\frac{P^{\prime}}{P}(z)\right|+\left|\frac{\psi^{\prime}}{\psi}(z)\right|+\left|\frac{f^{\prime}}{f}(z)\right| .
$$

Since $P$ is a polynomial, it is clear that

$$
\left|\frac{P^{\prime}}{P}(z)\right|=O\left(r^{-1}\right) \quad(|z|=r)
$$

and it follows easily from (2.11) that

$$
\left|\frac{\psi^{\prime}}{\psi}(z)\right|=O(1) \quad(z \in \mathscr{D}) .
$$

To estimate the last term on the right-hand side of (4.4) note that since $f \in U_{2 p}$,

$$
f(z)=c e^{Q(z)} \Pi(z)
$$

where $c$ is a constant, $Q(z)$ is a real polynomial of degree $\leqslant 2 p+2$, and $\Pi(z)$ is a canonical product of genus $\leqslant 2 p+1$ (recall the assumption from $\S 2$ that $f(0) \neq 0$ ). Thus Lemma 2 and (4.7) imply that

$$
\left|\frac{f^{\prime}}{f}(z)\right|=O\left(r^{2 p+1}\right) \quad(|z|=r, z \in \mathscr{D}) .
$$

In view of $(4.4)-(4.8)$, it follows that

$$
\left|\frac{F^{\prime \prime}}{F^{\prime}}(z)\right|=O\left(r^{2 p+1}\right) \quad(|z|=r, z \in \mathscr{D})
$$

which is incompatible with (4.3). This establishes the desired contradiction in the case that $g(z)$ has genus $\sigma \geqslant 2$.

In case $g(z)$ has genus $\sigma=0$ or $1,(4.2)$ and Lemma 4 imply that

$$
\left|\frac{F^{\prime \prime}}{F^{\prime}}(i r)\right| \leqslant \frac{1}{r^{2}} \quad\left(r \geqslant r_{0}\right) \text {. }
$$

To obtain a contradiction to this estimate from (4.1), we first treat the case where $f \in U_{2 p}$ with $p \geqslant 1$. In this situation, by (2.5) and the remarks preceding Lemma 1 , we have in particular that for all $r>0$,

$$
\left(f^{\prime} / f\right)(\text { ir })=P(\text { ir }) \psi(\text { ir })
$$

where $P$ is a polynomial of degree at least $2 p$. Since $\psi$ is holomorphic in the upper half plane and maps this region into the upper half plane, we also have by the Carathéodory inequality $[4, \mathrm{p} .18]$,

$$
A_{1} / r<|\psi(i r)|<A_{2} r \quad(r>0),
$$

where $A_{1}$ and $A_{2}$ are positive constants independent of $r$. We see, thus, that

$$
\left|\left(f^{\prime} / f\right)(i r)\right| \geqslant A r^{2 p-1} \quad\left(r \geqslant r_{0}, A>0\right) .
$$

It now follows readily from (4.1), Lemma 5, (4.5), and (4.13), that for $p \geqslant 1$ 


$$
\left|\left(F^{\prime \prime} / F^{\prime}\right)(i r)\right| \neq o(1 / r)
$$

which contradicts (4.10).

If on the other hand, $f \in U_{0}$, then

$$
\left(f^{\prime} / f\right)(z)=\alpha z+\beta+\left(\Pi^{\prime} / \Pi\right)(z),
$$

where $\alpha$ and $\beta$ are real and $\Pi$ is the canonical product of the zeros of $f$ and has genus 0 or 1 .

For $\alpha \neq 0$, Lemma 2 and (4.15) imply that

$$
\mid\left(f^{\prime} / f\right)(\text { ir }) \mid \geqslant(|\alpha| / 2) r \quad\left(r \geqslant r_{0}\right),
$$

and from (4.1), (4.5), and (4.6) we again have a contradiction to (4.10).

If $\alpha=0$, then

$$
\frac{f^{\prime}}{f}(z)=\beta+\frac{\Pi^{\prime}}{\Pi}(z)=\beta+z^{p_{1}} \sum_{k} \frac{m_{k}}{a_{k}^{p_{1}}\left(z-a_{k}\right)}
$$

where $p_{1}=$ genus of $\Pi(z)=0$ or 1 , and $m_{k}=$ multiplicity of the zero $a_{k}$. From (4.17) it follows that

$$
\left|\frac{f^{\prime}}{f}(i r)\right| \geqslant\left|\operatorname{Im} \frac{f^{\prime}}{f}(i r)\right|=r \sum_{k} \frac{m_{k}}{r^{2}+a_{k}^{2}} \geqslant r \sum_{\left|a_{k}\right|<r} \frac{m_{k}}{r^{2}+a_{k}^{2}} \geqslant \frac{1}{2} \frac{n(r)}{r}
$$

where $n(r)$ denotes the number of zeros of $f(z)$ (counting multiplicity) in the disc $|z| \leqslant r$ (i.e., in the interval $[-r, r]$ since the zeros of $f$ are real). Since $f$ has an infinite number of zeros, (4.18) implies that

$$
\liminf _{r \rightarrow \infty} r \mid\left(f^{\prime} / f\right)(\text { ir }) \mid=\infty \text {. }
$$

Combining (4.19) with (4.5) and Lemma 5 in (4.1) we see that

$$
\left|\frac{F^{\prime \prime}}{F^{\prime}}(i r)\right| \geqslant \frac{1}{r} \quad\left(r \geqslant r_{1}\right) \text {. }
$$

Since this gives the contradiction to $(4.10)$ in this final case, the proof is now complete.

\section{REFERENCES}

1. S. Hellerstein and J. Williamson, Derivatives of entire functions and a question of Pólya, Trans. Amer. Math. Soc. 227 (1977), 227-249.

2. Derivatives of entire functions and a question of Pólya. II, Trans. Amer. Math. Soc. 234 (1977), 497-503.

3. E. Laguerre, Sur les fonctions du genre zéro et du genre un, C. R. Acad. Sci. Paris Sér. A-B 98 (1882); Oeuvres 1 (1898), 174-177.

4. B. Ja. Levin, Distribution of zeros of entire functions, Transl. Math. Monographs, vol. 5, Amer. Math. Soc., Providence, R. I., 1964.

5. B. Ja. Levin and I. V. Ostrovskii, The dependence of the growth of an entire function on the distribution of the zeros of its derivatives, Amer. Math. Soc. Transl. (2) 32 (1963), 322-357.

6. G. Pólya, Über Annaherung durch Polynome mit lauter reellen Wurzeln, Rend. Circ. Mat. Palermo (2) 36 (1913), 279-295.

7. G. Valiron, Sur les fonctions entières d'ordre fini et d'ordre nul, et en particulier les fonctions $\dot{a}$ correspondance régulière, Ann. Fac. Sci. Univ. Toulsouse (3) 5 (1913), 117-257.

Department of Mathematics, University of Wisconsin, Madison, Wisconsin 53706

Department of Mathematics, University of Hawail, Honolulu, Hawail 96822 